\title{
Acute kidney injury in solitary kidney patients after partial nephrectomy: incidence, risk factors and prediction
}

\author{
Kun Zhu ${ }^{1,2,3 \#}$, Haifeng Song ${ }^{1,2,3}$, Zhenan Zhang ${ }^{1,2,3}$, Binglei Ma ${ }^{1,2,3}$, Xiaoyuan Bao ${ }^{4}$, Qian Zhang ${ }^{1,2,3}$ Jie Jin $^{1,2,3}$ \\ ${ }^{1}$ Department of Urology, Peking University First Hospital, Beijing 100034, China; ${ }^{2}$ Institute of Urology, Peking University, Beijing 100034, China; \\ ${ }^{3}$ National Research Center for Genitourinary Oncology, Beijing 100034, China; ${ }^{4}$ Medical Informatics Center, Peking University Health Science \\ Center, Beijing 100191, China \\ Contributions: (I) Conception and design: K Zhu, H Song, Q Zhang; (II) Administrative support: None; (III) Provision of study materials or patients: \\ K Zhu, H Song; (IV) Collection and assembly of data: K Zhu, X Bao; (V) Data analysis and interpretation: K Zhu, X Bao, B Ma, Z Zhang; (VI) \\ Manuscript writing: All authors; (VII) Final approval of manuscript: All authors. \\ \#These authors contributed equally to this work. \\ Correspondence to: Prof. Qian Zhang. Department of Urology, Peking University First Hospital, 8 Xishiku Street, Xicheng District, Beijing 100034, \\ China. Email: zhangqianbjmu@126.com.
}

Background: To analyze the incidence and risk factors of acute kidney injury (AKI) after partial nephrectomy $(\mathrm{PN})$ in patients with solitary kidney, and to build AKI prediction models using logistic regression and machine learning (ML) approaches.

Methods: Clinical data of 87 solitary kidney patients with renal mass who received PN from January 2003 to March 2019 were collected. The diagnosis of AKI was based on KDIGO criteria. Logistic regression analysis and ML method were used to build prediction models.

Results: AKI developed in $52(59.8 \%)$ patients. The logistic regression model had three variables: ischemia time $(\mathrm{P}=0.003)$, surgery time $(\mathrm{P}=0.001)$ and preoperative fasted blood glucose level $(\mathrm{FBG})(\mathrm{P}=0.049)$. The area under curve (AUC) was 0.826 , with the specificity and sensitivity of optimal threshold value $82.9 \%$ and 69.2\%. The ML model had the following variables: ischemia time, surgery time, age, FBG, mean arterial pressure (MAP), colloid, crystalloid, etc. XGBoost model has the best prediction performance. The AUC was 0.749 , lower than that of the logistic regression model with no statistical difference $(\mathrm{P}=0.258)$, with the specificity and sensitivity $62.9 \%$ and $84.6 \%$, respectively.

Conclusions: The incidence of AKI after PN in patients with a solitary kidney was relatively high, it was associated with longer ischemia time, surgery time and higher FBG level, etc. The performance of ML model had no significant difference with logistic regression model. Prospective studies with larger sample sizes are awaited to test and verify our research findings.

Keywords: Acute kidney injury (AKI); machine learning (ML); partial nephrectomy (PN); prediction model; solitary kidney

Submitted Nov 20, 2019. Accepted for publication Mar 16, 2020.

doi: $10.21037 /$ tau.2020.03.45

View this article at: http://dx.doi.org/10.21037/tau.2020.03.45

\section{Introduction}

It's not unusual for patients undergoing partial nephrectomy (PN) or radical nephrectomy to develop acute kidney injury (AKI) $(1,2)$. AKI might hurt long-term renal function and increases the incidence of cardiovascular accident and mortality, perioperatively $(3,4)$. Preventive strategies such as early identification and timely interventions could potentially reduce postoperative AKI and thereby improve prognosis dramatically $(5,6)$. A series of studies had found that several novel biomarkers such as cystatin $\mathrm{C}$ and neutrophil gelatinase-associated lipocalin (NGAL) could be used for detecting AKI after PN early and there were some 
approaches to measure biomarkers isolated from only the affected kidney before, during and after surgery to increase the sensitivity $(7,8)$. But the research of prediction models is rather little.

Tumors in a solitary kidney represent an imperative indication for PN rather than radical nephrectomy. For these patients, PN could preserve renal function, reduce the incidence of complications as possible while optimizing oncologic outcomes (9-11). Risk factors leading to AKI after $\mathrm{PN}$ in patients with a solitary kidney remain controversial. Finding possible risk factors and build reliable prediction models appear to be particularly important (12).

Machine learning (ML) approaches have been applied in medical fields as an emerging technological means, such as imaging diagnosis, pharmaceutical research and the establishment of prediction model $(13,14)$. They were reported to apply to build AKI prediction models of patients endure cardiac surgery and liver transplantation $(15,16)$. This research aims to investigate the incidence and risk factors of AKI in patients with a solitary kidney who underwent $\mathrm{PN}$ and to establish AKI prediction models using ML technologies and traditional logistic regression models, allowing more discerning prediction and assessment of AKI after PN.

\section{Methods}

\section{Study population}

From January 2003 to March 2019, 87 consecutive patients with a solitary kidney who were diagnosed with renal masses and treated with PN were included in this study. Inclusion criteria was patients with anatomical or functional solitary kidney. Functional solitary kidney was defined as unilateral kidney atrophy with poor renal function, while the functioning one suffering tumors. Exclusion criteria includes incomplete surgery, receiving renal replacement therapy such as hemodialysis and renal transplantation before surgery and missing key clinical records. This study was in compliance with the Helsinki declaration and was conducted after approval of the Institutional Ethical Review Board of Peking University First Hospital \{approval number: 2019[238]\}. Written informed consent was waived because of the retrospective nature of this study. All data were maintained with confidentiality.

\section{Data collecting}

Recorded variables included clinical, perioperative, pathological and laboratory data. All patients were examined by preoperative ultrasound, contrast enhanced computed tomography (CT) or magnetic resonance imaging (MRI). Three of them underwent contrast enhanced computed tomography. The clinical variables recorded included age at PN, sex, comorbidity, premedication, tobacco and alcohol addiction, nutritional status, tumor size, R.E.N.A.L. score, etc. Perioperative data studied included surgery and ischemia time, mean arterial pressure (MAP), colloid, crystalloid, total fluid, estimated blood loss, urine output, units transfused (includes packed red blood cell and fresh frozen plasma), anaesthetic gas, amount of post-operation drainage, hospital stays. Pathological features included histological subtype, tumor size, tumor stage, nuclear grade and multifocality. Laboratory results included blood urea nitrogen (BUN), serum potassium concentration $\left(\mathrm{K}^{+}\right)$, serum albumin (ALB), hemoglobin $(\mathrm{Hb})$, serum creatinine (SCr) and fasted blood glucose (FBG) perioperatively. R.E.N.A.L. score was used to help the decision of surgery approach before surgery (17). The approach depended on the following factors: the proficiency of surgeon, clinical tumor stage, $\mathrm{T} 2$ and above tumors were recommended open approach, the R.E.N.A.L. score, the score of 10 and above tumors were recommended open approach. Open surgery was through abdominal or lumbar incision, laparoscopic surgery was through intraperitoneal or retroperitoneal approach. As for the pattern of resection techniques during $\mathrm{PN}$, it depended on the size and location of tumor and the experience of surgeon. These strategies included three categories: enucleation, enucleoresection and resection (18-21). The strategy used most was resection. Ischemia type depended on the willing of surgeon and estimation of ischemia time. In general, cold ischemia has been used in surgery for those cases where the clamping time was expected more than 30 minutes. Anaesthesia was induced using $1.0-1.5 \mathrm{mg} / \mathrm{kg}$ propofol or $0.3 \mathrm{mg} / \mathrm{kg}$ etomidate, $0.3 \mu \mathrm{g} / \mathrm{kg}$ sufentanyl and $0.6 \mathrm{mg} / \mathrm{kg}$ rocuronium. Anesthesia was maintained with oxygen, air, sevoflurane, propofol 15 $35 \mathrm{~mL} / \mathrm{h}$, remifentanil (TCI) $2-3 \mathrm{ng} / \mathrm{mL}$, and cisatracurium was used for muscle relaxant. All patients were monitored by routine electrocardiography, pulse oximetry and noninvasive blood pressure, capnography, arterial blood pressure, BIS index and urine output. Crystalloid (Lactated Ringer's solution or $0.9 \%$ sodium chloride injection) and colloid (hetastarch or succinylated Gelatin) solutions were administered, and blood transfusions were used when needed.

The diagnosis of AKI is based on KDIGO criteria. The 
KDIGO criteria defined AKI as follows: increase in serum creatinine by $\geq 0.3 \mathrm{mg} / \mathrm{dL}(\geq 26.5 \mathrm{mmol} / \mathrm{L})$ within 48 hours, or increase in serum creatinine to $\geq 1.5$ times baseline, which was known or presumed to have occurred within the prior seven days after surgery, or urine volume $<0.5 \mathrm{~mL} / \mathrm{kg} / \mathrm{hour}$ for six hours (5). Estimated glomerular filtration rate (eGFR) was calculated using the Chronic Kidney Disease Epidemiology Collaboration (CKD-EPI) equation.

\section{Statistical analysis}

Continuous variables were compared using Student $t$-test and Mann-Whitney test while categorical variables were compared using Chi-square and Fisher's exact tests. Logistic regression was used for univariate and multivariable analysis for predictive factors for AKI. ML model establishments and internal validation was performed using the leave-one- out cross validation (LOOCV) using 5 algorithms: logistic regression (LR), support vector machines (SVM), decision tree (DT), random forest (RF) and XGBoost. Z test was used to compare the significance of difference of AUC of the prediction models. All $\mathrm{P}$ values were two-tail and $\mathrm{P}<0.05$ was considered significant. Data were analyzed using SPSS version 24.0 (SPSS Inc., Chicago, IL, USA) and R version 3.6.1 (R Foundation for Statistical Computing, Vienna, Austria) was used to build the ML prediction models.

\section{Results}

\section{Perioperative data}

A total of 87 patients with a solitary kidney underwent PN met the study criteria and were included in this analysis (Table 1). According to postoperative pathological reports,

Table 1 Patients and tumor characteristics, association between AKI and modifiable factors in single factor analysis

\begin{tabular}{|c|c|c|c|c|}
\hline Factors & Overall $(n=87)$ & AKI $(n=52)$ & No AKI $(n=35)$ & $P$ value \\
\hline Age (years) & $59.69 \pm 10.19$ & $61.33 \pm 9.08$ & $57.26 \pm 11.34$ & 0.081 \\
\hline Male & $66(75.90 \%)$ & $41(78.80 \%)$ & $25(71.40 \%)$ & 0.428 \\
\hline Female & $21(24.10 \%)$ & $11(21.2 \%)$ & $10(28.6 \%)$ & 0.428 \\
\hline Tobacco use & $24(27.60 \%)$ & $15(28.80 \%)$ & $9(25.70 \%)$ & 0.749 \\
\hline Alcohol use & $21(24.10 \%)$ & $13(25.00 \%)$ & $8(22.90 \%)$ & 0.819 \\
\hline \multicolumn{5}{|l|}{ Preoperative factors } \\
\hline Hypertension & $36(41.40 \%)$ & $22(42.30 \%)$ & $14(40.00 \%)$ & 0.830 \\
\hline $\mathrm{FBG}(\mathrm{mmol} / \mathrm{L})$ & $5.42 \pm 1.07$ & $5.57 \pm 1.15$ & $5.18 \pm 0.92$ & 0.092 \\
\hline CKD & 26 (34.20\%) & $14(32.60 \%)$ & $12(34.29 \%)$ & 0.729 \\
\hline $\mathrm{SCr}(\mu \mathrm{mol} / \mathrm{L})$ & $102.13(88.00,116.00)$ & $103.16(91.67,115.49)$ & $101.87(84.74,116.26)$ & 0.559 \\
\hline eGFR $\left(\mathrm{mL} / \mathrm{min} / 1.73 \mathrm{~m}^{2}\right)$ & $64.77 \pm 16.11$ & $65.03 \pm 17.92$ & $64.42 \pm 13.65$ & 0.872 \\
\hline $\mathrm{Hb}(\mathrm{g} / \mathrm{L})$ & $140.31 \pm 18.83$ & $136.87 \pm 20.37$ & $145.42 \pm 15.14$ & $0.037^{\star}$ \\
\hline ALB $(g / L)$ & $42.49 \pm 4.22$ & $41.81 \pm 4.40$ & $43.50 \pm 3.78$ & 0.066 \\
\hline $\mathrm{K}^{+}(\mathrm{mmol} / \mathrm{L})$ & $4.00 \pm 0.36$ & $4.00 \pm 0.38$ & $4.01 \pm 0.32$ & 0.868 \\
\hline BUN (mmol/L) & $6.41(5.35,7.76)$ & $6.66(5.35,7.77)$ & $6.07(5.26,7.68)$ & 0.542 \\
\hline
\end{tabular}

Table 1 (continued) 
Table 1 (continued)

\begin{tabular}{|c|c|c|c|c|}
\hline Factors & Overall $(n=87)$ & AKI $(n=52)$ & No AKI (n=35) & $P$ value \\
\hline $\mathrm{CCl}$ & $3.00(1.00,4.00)$ & $3.00(1.00,4.00)$ & $3.00(1.00,4.00)$ & 0.892 \\
\hline \multicolumn{5}{|l|}{ Intraoperative factors } \\
\hline \multicolumn{5}{|l|}{ Surgical approach } \\
\hline Laparoscopic & $37(42.50 \%)$ & $16(30.80 \%)$ & $21(60.00 \%)$ & $0.007^{*}$ \\
\hline Clamping & $79(90.80 \%)$ & $49(94.23 \%)$ & $30(85.71 \%)$ & \multirow[t]{2}{*}{0.178} \\
\hline Off clamping & $8(9.20 \%)$ & $3(5.77 \%)$ & $5(14.29 \%)$ & \\
\hline Surgery time (min) & $115.00(82.00,162.00)$ & $150.00(105.50,178.50)$ & $100.00(58.00,122.00)$ & $<0.001^{\star}$ \\
\hline Warm & $39(49.37 \%)$ & $22(44.90 \%)$ & 17 (56.67\%) & \multirow[t]{2}{*}{0.310} \\
\hline Cold & $40(50.63 \%)$ & $27(55.10 \%)$ & $13(43.33 \%)$ & \\
\hline Warm ischemia time (min) & $19(15,27)$ & $23(18,36)$ & $15(13,19)$ & $0.003^{*}$ \\
\hline Cold ischemia time (min) & $20(15,29)$ & $21(20,32)$ & $18(13,23)$ & $0.045^{*}$ \\
\hline \multicolumn{5}{|l|}{ Intraoperative fluid } \\
\hline Crystalloid (mL) & $1600.00(1125.00,1825.00)$ & $1622.50(1225.00,1837.50)$ & $1425.00(1100.00,1825.00)$ & 0.135 \\
\hline Colloid (mL) & $0.00(0.00,500.00)$ & $0.00(0.00,500.00)$ & $0.00(0.00,0.00)$ & $0.049^{\star}$ \\
\hline Total fluid (mL) & $1700.00(1225.00,2200.00)$ & $1737.50(1500.00,2243.75)$ & $1600.00(1100.00,2200.00)$ & 0.050 \\
\hline \multicolumn{5}{|l|}{ Pathological tumor stage } \\
\hline Benign & $9(10.24 \%)$ & $4(7.70 \%)$ & $5(14.29 \%)$ & $0.322^{\ddagger}$ \\
\hline Multifocality & $19(21.80 \%)$ & $14(26.90 \%)$ & $5(14.30 \%)$ & 0.162 \\
\hline Post-operation RRT & $4(4.60 \%)$ & 4 (7.70\%) & 0 & 0.093 \\
\hline Hospital stays (d) & $10.00(7.00,13.00)$ & $11.00(8.00,16.00)$ & $7.00(6.00,9.00)$ & $<0.001^{\star}$ \\
\hline Admission to ICU & $19(21.84 \%)$ & $18(34.62 \%)$ & $1(2.86 \%)$ & $<0.001^{*}$ \\
\hline ICU stays (d) & $0.00(0.00,0.00)$ & $0.00(0.00,1.00)$ & $0.00(0.00,0.00)$ & $0.001^{*}$ \\
\hline
\end{tabular}

Continuous variables are presented as medians with quartiles or means with standard deviation. Frequencies with proportions are displayed for categorical variables. The number in parenthesis represents the number of patients with available data. ${ }^{*} \mathrm{P}<0.05 ;{ }^{\dagger}$ represented comparison pathological T1,T2 with T3,T4 malignant tumor; ${ }^{\ddagger}$ represented comparison malignant with benign tumor. AKI, acute kidney injury; BMI, body mass index; MAP, mean arterial pressure; FBG, fasted blood glucose; CKD, chronic kidney disease; SCr, serum creatinine; eGFR, estimated glomerular function rate; Hb, hemoglobin; ALB, albumin; BUN, blood urea nitrogen; CCI, Charlson comorbidity index; $\mathrm{RRT}$, renal replacement therapy; ICU, intensive care unit. 
excised tumors were renal cell carcinoma in the majority of cases (78 or $89.66 \%$ ), oncocytoma in 2, angiomyolipoma in 5 , cavernous hemangioma in 1 and benign cysts in 1 .

Fifty-two patients (59.77\%) were diagnosed with AKI after PN. Thirty-two patients $(36.78 \%$ ) had AKI (grade 1), 10 (11.49\%) had grade 2 and 10 (11.49\%) had grade 3. Postoperative renal failure occurred in 4 patients $(4.60 \%)$ who subsequently received renal replacement therapy (RRT).

With regard to preoperative factors, patients with AKI and non-AKI differed in a statistically significant according to $\mathrm{Hb}(\mathrm{P}=0.037)$, tumor diameter $(\mathrm{P}=0.017)$, R.E.N.A.L score $(\mathrm{P}=0.024)$. As for intraoperative characters, postoperative AKI were significantly associated with open surgery $(\mathrm{P}=0.007)$, longer surgical $(\mathrm{P}<0.001)$ and ischemia time $(\mathrm{P}<0.001)$, colloid $(\mathrm{P}=0.049)$, longer hospital duration $(\mathrm{P}<0.001)$, postoperative intensive care unit (ICU) admission $(\mathrm{P}<0.001)$ and longer ICU duration $(\mathrm{P}=0.001)$.

\section{Subgroup analysis of CKD group}

In CKD group, patients with $\mathrm{AKI}$ and non-AKI differed in a statistically significant according to $\mathrm{Hb}(\mathrm{P}=0.006)$, ALB $(\mathrm{P}=0.014)$, surgery approach $(\mathrm{P}=0.006)$, surgical $(\mathrm{P}=0.004)$ and ischemia time $(\mathrm{P}=0.036)$ (Table 2$)$.

Table 2 Subgroup analysis of CKD group

\begin{tabular}{|c|c|c|c|c|}
\hline Factors & Overall $(n=26)$ & AKI $(n=14)$ & No AKI $(n=12)$ & $P$ value \\
\hline Age (years) & $67.46 \pm 8.10$ & $68.21 \pm 7.81$ & $66.58 \pm 8.70$ & 0.619 \\
\hline Male & $22(84.62 \%)$ & $13(92.86 \%)$ & $9(75.00 \%)$ & 0.208 \\
\hline Female & $4(15.38 \%)$ & $1(7.14 \%)$ & $3(25.00 \%)$ & \\
\hline Tobacco use & $8(30.77 \%)$ & $5(35.71 \%)$ & $3(25.00 \%)$ & 0.555 \\
\hline Alcohol use & $4(15.38 \%)$ & $2(14.29 \%)$ & $2(16.67 \%)$ & 0.867 \\
\hline \multicolumn{5}{|l|}{ Preoperative factors } \\
\hline Hypertension & $13(50.00 \%)$ & $8(57.14 \%)$ & $5(41.67 \%)$ & 0.431 \\
\hline FBG (mmol/L) & $5.55 \pm 1.39$ & $5.66 \pm 1.71$ & $5.43 \pm 0.97$ & 0.685 \\
\hline $\mathrm{SCr}(\mu \mathrm{mol} / \mathrm{L})$ & $124.50(113.74,138.38)$ & $124.50(116.26,147.00)$ & $120.75(110.64,138.61)$ & 0.432 \\
\hline eGFR $\left(\mathrm{mL} / \mathrm{min} / 1.73 \mathrm{~m}^{2}\right)$ & $47.63 \pm 9.96$ & $45.39 \pm 12.23$ & $50.24 \pm 5.89$ & 0.223 \\
\hline $\mathrm{Hb}(\mathrm{g} / \mathrm{L})$ & $132.27 \pm 17.94$ & $123.79 \pm 18.28$ & $142.17 \pm 11.76$ & $0.006^{*}$ \\
\hline ALB (g/L) & $41.05 \pm 4.08$ & $39.29 \pm 4.11$ & $43.12 \pm 3.05$ & $0.014^{*}$ \\
\hline $\mathrm{K}^{+}(\mathrm{mmol} / \mathrm{L})$ & $4.09 \pm 0.46$ & $4.11 \pm 0.54$ & $4.07 \pm 0.37$ & 0.847 \\
\hline BUN (mmol/L) & $7.69(6.50,8.60)$ & $6.66(5.35,7.77)$ & $6.07(5.26,7.68)$ & 0.820 \\
\hline R.E.N.A.L score & $7.00(6.00,8.25)$ & $4.00(2.00,6.25)$ & $5.00(3.00,6.75)$ & 0.095 \\
\hline
\end{tabular}

Table 2 (continued) 
Table 2 (continued)

\begin{tabular}{|c|c|c|c|c|}
\hline Factors & Overall $(n=26)$ & AKI $(n=14)$ & No AKI $(n=12)$ & $P$ value \\
\hline Ischemia time (min) & $20.00(15.00,23.25)$ & $20.50(19.50,25.25)$ & $15.00(8.50,21.25)$ & $0.036^{*}$ \\
\hline \multicolumn{5}{|l|}{ Type of ischemia } \\
\hline Warm & $1246.15 \%)$ & $6(42.86 \%)$ & $6(50.00 \%)$ & 0.561 \\
\hline \multicolumn{5}{|l|}{ Intraoperative fluid } \\
\hline Crystalloid (mL) & $1225.00(1100.00,1762.50)$ & $1412.50(1225.00,1762.50)$ & $1100.00(912.50,1925.00)$ & 0.060 \\
\hline Colloid (mL) & $0.00(0.00,0.00)$ & $0.00(0.00,500.00)$ & $0.00(0.00,0.00)$ & 0.131 \\
\hline Total fluid (mL) & $1600.00(1100.00,1812.50)$ & $1672.50(1225.00,1862.50)$ & $1100.00(912.50,1925.00)$ & 0.060 \\
\hline Tumor maximum diameter $(\mathrm{cm})$ & $3.07(1.82,4.02)$ & $3.41(1.86,5.70)$ & $2.62(1.66,3.21)$ & 0.087 \\
\hline Multifocality & $7(26.92 \%)$ & $4(28.57 \%)$ & $3(25.00 \%)$ & 0.838 \\
\hline
\end{tabular}

Continuous variables are presented as medians with quartiles or means with standard deviation. Frequencies with proportions are displayed for categorical variables. The number in parenthesis represents the number of patients with available data. ${ }^{*} \mathrm{P}<0.05$. $\mathrm{BMI}$, body mass index; MAP, mean arterial pressure; FBG, fasted blood glucose; CKD, chronic kidney disease; SCr, serum creatinine; eGFR, estimated glomerular function rate; Hb, hemoglobin; ALB, albumin; BUN, blood urea nitrogen; CCI, Charlson comorbidity index; RRT, renal replacement therapy; ICU, intensive care unit.

Table 3 Multivariable logistic regression analysis of factors for AKI after PN

\begin{tabular}{|c|c|c|c|c|c|}
\hline \multirow{2}{*}{ Risk factors } & \multirow{2}{*}{ Coefficient } & \multirow{2}{*}{ Odds ratio } & \multicolumn{2}{|c|}{$95 \% \mathrm{Cl}$} & \multirow{2}{*}{$P$ value } \\
\hline & & & $\mathrm{LCl}$ & $\mathrm{UCl}$ & \\
\hline Ischemia time & 0.092 & 1.096 & 1.031 & 1.165 & $0.003^{*}$ \\
\hline Surgery time & 0.018 & 1.018 & 1.007 & 1.029 & $0.001^{*}$ \\
\hline FBG & 0.640 & 1.896 & 1.001 & 3.590 & $0.049^{*}$ \\
\hline
\end{tabular}

Variables with $\mathrm{P}<0.1$ in single factor analysis and those with possible clinical significance were brought into multivariable logistic regression analysis using a cutoff of $\mathrm{P}$ value of less than 0.10 . The logistic regression analysis was established based on Forward LR. *, P<0.05. AKI, acute kidney injury; PN, partial nephrectomy; FBG, fasted blood glucose; Cl, confidence interval; LCl, lower confidence interval; UCl, upper confidence interval.

\section{Screening results of variables of the AKI prediction models}

According to the result of single-factor analysis shown in Table $1(\mathrm{P}<0.1$ was considered partly significant and these variables were chosen) and possible clinical significance, screening factors for prediction models were as follows: age, BMI, Surgery time, Ischemia time, MAP, BUN, $\mathrm{K}^{+}, \mathrm{ALB}$, FBG, HB, SCr, eGFR, crystalloid, colloid, total fluid, type of ischemia, RENAL score, tumor size, multifocality, etc.

\section{Establishment of the logistic regression prediction model}

The logistic regression prediction model had three variables: surgery time, ischemia time, FBG (Table 3). The model equation is Logit $\mathrm{P}=-6.919+0.018 \times$ surgery time $+0.092 \times$ ischemia time $+0.640 \times$ FBG. The AUC of the logistic regression prediction model was 0.826 (95\% CI: $0.740-0.912$ ) (Figure 1), with the specificity and sensitivity of optimal threshold value $82.9 \%$ and $69.2 \%$, respectively. 


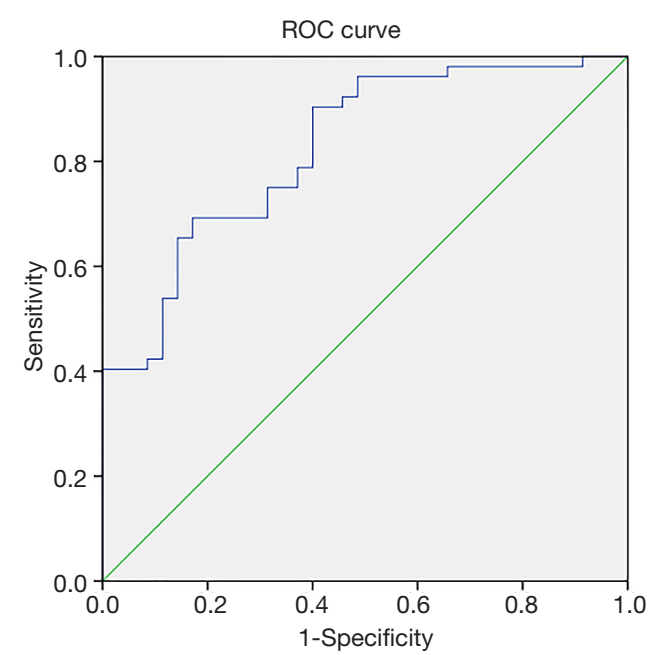

Figure 1 ROC of the logistic regression prediction model. ROC, the receiver operating characteristic curve.
The accuracy of the model was $74.7 \%$. Variables from the logistic regression model were utilized to build a nomogram for the prediction of AKI (Figure 2).

\section{Establishment of ML prediction model}

Model establishments and internal validation was performed using the leave-one-out cross validation (LOOCV), the precision, recall, F1 and AUC were calculated (Table 4). The result showed that XGBoost has the largest AUC and highest accuracy (Figure 3). The XGBoost prediction model had following variables: ischemia time, surgery time, BMI, age, BUN, $\mathrm{K}^{+}, \mathrm{MAP}$, crystalloid, ALB, multifocality, total fluid, FBG, $\mathrm{Hb}$ and R.E.N.A.L score.

The AUC of XGBoost prediction model was 0.749 (95\% CI: 0.648-0.851), slightly lower than the logistic regression

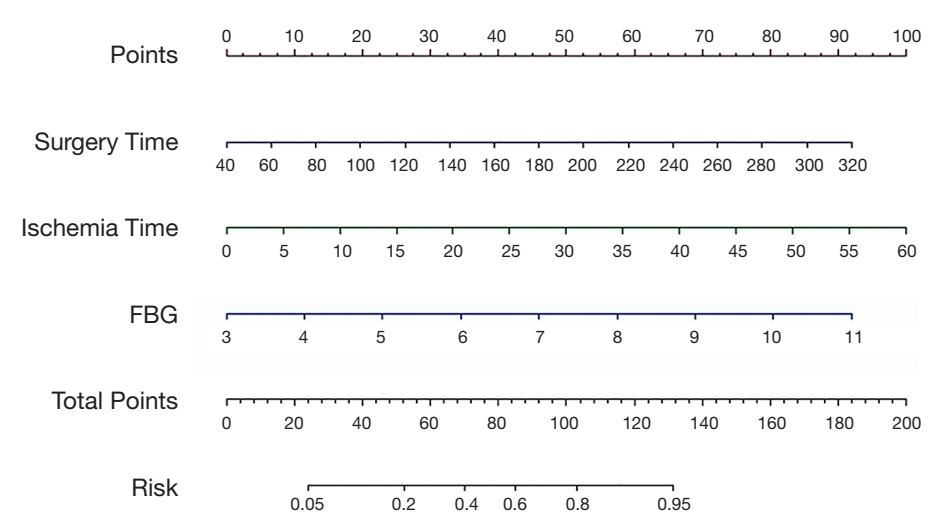

Figure 2 Nomogram for the prediction of AKI after PN based on logistic regression model. Instructions: locate the surgery time on the corresponding axis. Draw a line straight upward to the points axis to determine how many points toward the probability of AKI receives for his/her surgery time. Repeat the process for each variable. Add the points for each of variable. Locate the final points on the total points axis. Draw a line straight down to find the patient's probability of AKI after PN. AKI, acute kidney injury; PN, partial nephrectomy; FBG, fasted blood glucose.

Table 4 The prediction performance of five machine learning models

\begin{tabular}{lcccccc}
\hline \multirow{2}{*}{ Machine learning } & Precision & Recall & F1 & AUC & \multicolumn{2}{c}{$95 \% \mathrm{Cl}$} \\
\cline { 5 - 7 } & & & & & Lower limit & Upper limit \\
DT & 0.559 & 0.635 & 0.595 & 0.530 & 0.406 & 0.653 \\
RF & 0.667 & 0.769 & 0.714 & 0.718 & 0.611 & 0.825 \\
LR & 0.692 & 0.692 & 0.692 & 0.615 & 0.493 & 0.736 \\
SVM & 0.660 & 0.673 & 0.667 & 0.559 & 0.685 & 0.648 \\
XGBOOST & 0.772 & 0.846 & 0.807 & 0.749 & & 0.851
\end{tabular}

DT, decision tree, RF, random forest, LR, logistic regression, SVM, support vector machines. AUC, area under the receiver operating characteristic; $\mathrm{Cl}$, confidence interval. 


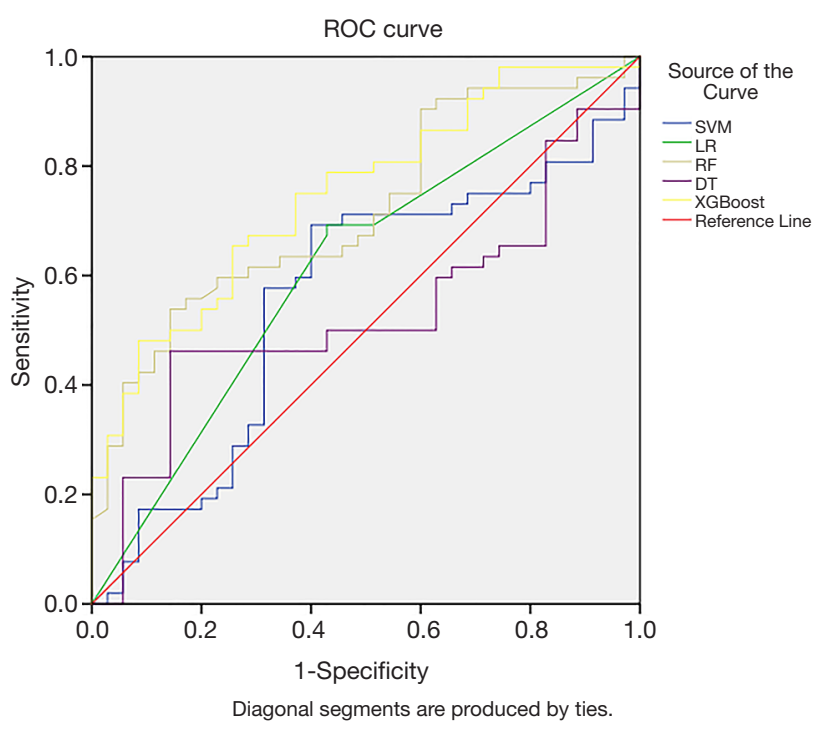

Figure 3 Comparison of area under the receiver operating characteristic curves among the machine learning models. ROC, the receiver operating characteristic curve; SVM, support vector machines; LR, logistic regression; DT, decision trees; RF, random forest.

model with no significant difference $(\mathrm{P}=0.258)$ with no significant difference, with the specificity and sensitivity of optimal threshold value $62.9 \%$ and $84.6 \%$, respectively. The accuracy of the model was $75.9 \%$.

\section{Discussion}

Patients with a solitary kidney were pretty good research subjects when studying the incidence and risk factors after PN (22). On the one hand, tumors in a solitary kidney represent an imperative indication for PN (23), on the other hand, the compensation from the contralateral healthy kidney of decreased kidney function was eliminated (24). Therefore, these patients were selected to find risk factors and build prediction models, providing new ideas for assessing the risk of postoperative AKI. As an emerging subject, ML had been applied in various medical fields. It was reported to be applied in building AKI prediction models of patients who endure cardiac surgery, liver transplantation and burn $(15,16,25)$. But limited studies were discussing AKI after PN using ML models. In this study, we built prediction models using $5 \mathrm{ML}$ algorithms and compared prediction ability with traditional logistic regression analysis.
In general, patients underwent assessment of SCr at the day after surgery and every other day. If patients were diagnosed as AKI, the assessment will increase to once a day and vice versa. In fact, the urinary output and liquid intake couldn't assess the renal function timely and accurately in our research. So, we diagnosed our patients using $\mathrm{SCr}$ and eGFR mostly. Our study showed that AKI developed in 52 (59.8\%) patients. According to the results of 2 prediction models, with regards to the preoperative risk factors, the levels of FBG, SCr, $\mathrm{K}^{+}$, BMI, ALB, BUN, Hb, MAP, age were associated with AKI after PN. As for intraoperative factors, surgery time, ischemia time, the number of crystalloids and total fluid volume as well as tumor multifocality and R.E.N.A.L score affected kidney function potentially. FBG, ischemia time and surgery time were included in both prediction models. We also performed a subgroup analysis of $\mathrm{CKD}$, in which consistent results were obtained. The performance of ML model had no significant difference with logistic regression model $(\mathrm{P}=0.258)$.

Patients with a solitary kidney were at high risk of AKI after PN. The incidence of AKI in patients with a solitary kidney was reported as about $54 \%$ while that of patients with a functioning contralateral kidney was $5-20.5 \%$ $(3,22,26)$. Our research showed $59.8 \%$ patients with a solitary kidney suffered AKI after PN in our medical center, slightly higher than other studies.

Previous studies have shown that ischemia time seemed to be strong factor associated with AKI, but there are still disputes about the influence of the type of ischemia on AKI after PN (27-29). Our study showed that ischemia time was the most important risk factors $(\mathrm{P}<0.001)$, the incidence rate of cold ischemia patients $(67.5 \%)$ exceeded that of warm ischemia patients (56.4\%). But the type of ischemia wasn't an independent risk factor. Now that the ischemia time is one of the most important risk factors of AKI, measures should be taken to reduce ischemia time regardless of the ischemia type (30).

In the study from Steven C. Campbell and his colleagues, warm ischemia $(\mathrm{P}=0.03)$ and diabetes $(\mathrm{P}=0.03)$ were associated with a long-term functional decline on multivariable analysis (12). So Kim and his colleagues had found that the incidence rate of postoperative AKI in patients after PN was significantly higher in patients with DM than in those without $\mathrm{DM}(30.7 \%$ vs. $14.9 \%, \mathrm{P}<0.001)$ (31). But another research found no significant relationship between FBG and AKI in patients with acute pancreatitis (32). In our study, a higher level of preoperative FBG means higher risk of AKI. It might be due to the fact that long-term high 
concentration glucose on glomerular mesangial cells and vascular smooth muscle cells led to high blood pressure, high transfusion and hyperfiltration of glomeruli $(1,33,34)$. Although patients hadn't developed renal insufficiency in early time, their poor abilities of tolerance to surgery and ischemia was associated with greater susceptibility of AKI. So, it appears to be particularly important of periodical monitoring and controlling for blood glucose.

In our single factor analysis, there were significant differences in the coincidence of AKI between open surgery and laparoscopic surgery group $(72.0 \%$ vs. $43.2 \%$, $\mathrm{P}=0.007)$. We still excluded surgery approach rom the logistic regression analysis. Although, there were significant differences between the two groups in surgery time [148.00 $(113.75,108)$ vs. 80.00 (55.00, 108.00) min, $\mathrm{P}<0.000]$, tumor maximum diameter $[3.54(2.30,4.80)$ vs. 2.60 (1.82, $3.68) \mathrm{cm}, \mathrm{P}=0.009]$, but no significance difference in ischemia time $[17.00(13.50,22.50) v s .20 .50(14.75,30.00)$ $\min , \mathrm{P}=0.179]$. It meant that patients with larger tumor and harder surgery possibility have greater probability of undergoing open surgery, longer surgery time and higher risk on AKI after PN. In the study from Brian R. Lane and his colleagues in 2008, the most important prediction factor of AKI was ischemia time rather than the type of surgery, which was generally consistent with our results (35).

Every minute counts when the renal hilum is clamped during PN (36). In our research, clamping or off clamping wasn't an independent risk factor $(\mathrm{P}=0.178)$, but anatomybased novel surgical approaches may reduce ischemic time during PN. In order to get minimizing ischemia and better visualization, a series of technologies such as early unclamping, segmental clamping, tumor-specific clamping (zero ischemia), and unclamped PN were studied (37-39).

Another interesting finding was that perioperative fluid balance might associate with postoperative renal function although we didn't look at other anaesthesia-related modifiable factors. High fluid intake especially crystalloid was found as a risk factor of AKI from our ML prediction model. In the study from Shen et al., postoperative positive fluid balance was associated with higher AKI incidence which was identical to our findings (40).

Our study showed that XGBoost has the largest AUC and best prediction performance of ML model, slightly worse than the logistic regression model, but there was no significant difference $(\mathrm{P}=0.258)$. XGBoost was an integrate ML system based on decision tree. XGBoost had superiorities of efficiency, flexibility and portability through a number of approaches such as out-of-core computation, cache-aware, sparsity-aware learning, shrinkage and column subsampling (41).

No evidence of superior performance of $M L$ over logistic regression had been presented previously (42), and our study proved that viewpoint. Some of the following factors could be related to this phenomenon: ML algorithms applied to large-scale data and multiple prediction factors. The amount of data required by the ML model was far more than that of logistic regression. The data of our retrospective cohort study couldn't meet the need of ML prediction model well. The ML models lacked of sufficient validation and promotion on account of the scarcity of training data. In addition, ML model exaggerated the role of extremely low and high factors such as surgery time and ischemia time possibly.

Our prediction models could be used for patient counseling and medical decision making. According to the probability calculated from our models, enhancement of monitoring, specific nursing and medical strategies were required after surgery for those patients at high risk.

Our study has certain limitations, including the following aspects: First, our analysis is a retrospective study with a small sample size, which means farther prospective studies and studies with larger sample capacity are needed to verify our results. Besides, the timeframe (16 years) evaluated is very wide, which makes our study less applicable. Second, surgeries were not performed by a single surgeon. Thus, differences in surgery experience and surgical skills, may lead to confounding bias to some degree. Third, objects of our study were patients with a solitary kidney. Previous studies showed that the solitary kidney is more likely in a state of hyperfiltration and it has more resistance compared with bilateral kidneys. Conclusions might be not entirely applicable for patients with bilateral kidneys. FBG was an independent risk factor in our study, but a single sampling appears not sufficient to make any inference on the glucose metabolism state. Our research adopted an anesthesia standardized protocol, and we didn't look at anaesthesiarelated modifiable factors, so there might be a potential bias. Besides, it needs long-term follow-up to find the relationship between perioperative risk factors and longterm renal function and build prediction models.

\section{Conclusions}

The incidence of AKI after PN in patients with a solitary kidney was relatively high, it was associated with longer ischemia time, surgery time and higher preoperative FBG, 
and had no significant correlation with surgery approach and ischemia type. The performance of ML model had no significant difference with logistic regression model. Both of them could predict AKI after PN satisfactorily. Prospective studies are expected to test and verify our research findings.

\section{Acknowledgments}

Funding: None.

\section{Footnote}

Conflicts of Interest: All authors have completed the ICMJE uniform disclosure form (available at http://dx.doi. org/10.21037/tau.2020.03.45). QZ serves as an unpaid editorial board member of Translational Andrology and Urology from May 2015 to Apr 2021. The other authors have no conflicts of interest to declare.

Ethical Statement: The authors are accountable for all aspects of the work in ensuring that questions related to the accuracy or integrity of any part of the work are appropriately investigated and resolved. This study was approved by the institutional ethics committee of Peking University First Hospital \{approval number: 2019[238]\}. Because it was a retrospective analysis of routine data, a waiver of wrote informed consent was granted from the ethics committee. Patient records or information were anonymous and de-identified prior to analysis.

Open Access Statement: This is an Open Access article distributed in accordance with the Creative Commons Attribution-NonCommercial-NoDerivs 4.0 International License (CC BY-NC-ND 4.0), which permits the noncommercial replication and distribution of the article with the strict proviso that no changes or edits are made and the original work is properly cited (including links to both the formal publication through the relevant DOI and the license). See: https://creativecommons.org/licenses/by-nc-nd/4.0/.

\section{References}

1. Rajan S, Babazade R, Govindarajan SR, et al. Perioperative factors associated with acute kidney injury after partial nephrectomy. Br J Anaesth 2016;116:70-6.

2. Schmid M, Krishna N, Ravi P, et al. Trends of acute kidney injury after radical or partial nephrectomy for renal cell carcinoma. Urol Oncol 2016;34:293.e1-e10.
3. Bravi CA, Vertosick E, Benfante N, et al. Impact of Acute Kidney Injury and Its Duration on Long-term Renal Function After Partial Nephrectomy. Eur Urol 2019;76:398-403.

4. Gameiro J, Fonseca JA, Neves M, et al. Acute kidney injury in major abdominal surgery: incidence, risk factors, pathogenesis and outcomes. Ann Intensive Care 2018;8:22.

5. Vanmassenhove J, Kielstein J, Jörres A, et al. Management of patients at risk of acute kidney injury. Lancet 2017;389:2139-51.

6. Mir MC, Ercole C, Takagi T, et al. Decline in Renal Function after Partial Nephrectomy: Etiology and Prevention. J Urol 2015;193:1889-98.

7. Lane BR, Babitz SK, Vlasakova K, et al. Evaluation of Urinary Renal Biomarkers for Early Prediction of Acute Kidney Injury Following Partial Nephrectomy: A Feasibility Study. Eur Urol Focus 2018. doi: 10.1016/ j.euf.2018.10.017.

8. Antonelli A, Allinovi M, Cocci A, et al. The Predictive Role of Biomarkers for the Detection of Acute Kidney Injury After Partial or Radical Nephrectomy: A Systematic Review of the Literature. Eur Urol Focus 2020;6:344-53.

9. Haber G-P, Gill IS. Laparoscopic Partial Nephrectomy: Contemporary Technique and Outcomes. Eur Urol 2006;49:660-5.

10. Van Poppel H, Da Pozzo L, Albrecht W, et al. A Prospective, Randomised EORTC Intergroup Phase 3 Study Comparing the Oncologic Outcome of Elective Nephron-Sparing Surgery and Radical Nephrectomy for Low-Stage Renal Cell Carcinoma. Eur Urol 2011;59:543-52.

11. MacLennan S, Imamura M, Lapitan MC, et al. Systematic Review of Perioperative and Quality-of-life Outcomes Following Surgical Management of Localised Renal Cancer. Eur Urol 2012;62:1097-117.

12. Zabell J, Isharwal S, Dong W, et al. Acute Kidney Injury after Partial Nephrectomy of Solitary Kidneys: Impact on Long-Term Stability of Renal Function. J Urol 2018;200:1295-301.

13. Rajkomar A, Dean J, Kohane I. Machine Learning in Medicine. N Engl J Med 2019;380:1347-58.

14. Chen PC, Liu Y, Peng L. How to develop machine learning models for healthcare. Nat Materials 2019;18:410-4.

15. Lee HC, Yoon SB, Yang SM, et al. Prediction of Acute Kidney Injury after Liver Transplantation: Machine Learning Approaches vs. Logistic Regression Model. J 
Clin Med 2018;7:428.

16. Huang C, Murugiah K, Mahajan S, et al. Enhancing the prediction of acute kidney injury risk after percutaneous coronary intervention using machine learning techniques: A retrospective cohort study. PLoS Med 2018; 15:e1002703.

17. Kutikov A, Uzzo RG. The R.E.N.A.L. nephrometry score: a comprehensive standardized system for quantitating renal tumor size, location and depth. J Urol 2009;182:844-53.

18. Minervini A, Campi R, Lane BR, et al. Impact of Resection Technique on Perioperative Outcomes and Surgical Margins after Partial Nephrectomy for Localized Renal Masses: A Prospective Multicenter Study. J Urol 2020;203:496-504.

19. Minervini A, Campi R, Sessa F, et al. Positive surgical margins and local recurrence after simple enucleation and standard partial nephrectomy for malignant renal tumors: systematic review of the literature and meta-analysis of prevalence. Minerva Urol Nefrol 2017;69:523-38.

20. Mari A, Tellini R, Di Maida F, et al. Predictors of early postoperative and mid-term functional outcomes in patients treated with Endoscopic Robot Assisted Simple Enucleation (ERASE): results from a tertiary referral centre. Minerva Urol Nefrol 2019. [Epub ahead of print].

21. Minervini A, Carini M, Uzzo RG, et al. Standardized reporting of resection technique during nephron-sparing surgery: the surface-intermediate-base margin score. Eur Urol 2014;66:803-5.

22. Zhang Z, Zhao J, Dong W, et al. Acute Kidney Injury after Partial Nephrectomy: Role of Parenchymal Mass Reduction and Ischemia and Impact on Subsequent Functional Recovery. Eur Urol 2016;69:745-52.

23. Ghavamian R, Cheville JC, Lohse CM, et al. Renal Cell Carcinoma in the Solitary Kidney: An Analysis of Complications and Outcome After Nephron Sparing Surgery. J Urol 2002;168:454-9.

24. Bertolo R, Capitanio U. Re: Acute Kidney Injury After Partial Nephrectomy in Solitary Kidneys: Impact on Longterm Stability of Renal Function. Eur Urol 2019;75:346-8.

25. Tran NK, Sen S, Palmieri TL, et al. Artificial intelligence and machine learning for predicting acute kidney injury in severely burned patients: A proof of concept. Burns 2019;45:1350-8.

26. Campbell SC, Novick AC, Streem SB, et al. Complications of Nephron Sparing Surgery for Renal Tumors. J Urol 1994;151:1177-80.

27. Volpe A, Blute ML, Ficarra V, et al. Renal Ischemia and
Function After Partial Nephrectomy: A Collaborative Review of the Literature. Eur Urol 2015;68:61-74.

28. Parekh DJ, Weinberg JM, Ercole B, et al. Tolerance of the human kidney to isolated controlled ischemia. J Am Soc Nephrol 2013;24:506-17.

29. Wahlberg E, DiMuzio PJ, Stoney RJ. Aortic clamping during elective operations for infrarenal disease: The influence of clamping time on renal function. J Vasc Surg 2002;36:13-8.

30. Thompson RH, Frank I, Lohse CM, et al. The Impact of Ischemia Time During Open Nephron Sparing Surgery on Solitary Kidneys: A Multi-Institutional Study. J Urol 2007;177:471-6.

31. Kim NY, Hong JH, Koh DH, et al. Effect of Diabetes Mellitus on Acute Kidney Injury after Minimally Invasive Partial Nephrectomy: A Case-Matched Retrospective Analysis. J Clin Med 2019. doi: 10.3390/jcm8040468.

32. Chai X, Huang HB, Feng G, et al. Baseline Serum Cystatin C Is a Potential Predictor for Acute Kidney Injury in Patients with Acute Pancreatitis. Dis Markers 2018;2018:8431219.

33. Shacham Y, Gal-Oz A, Leshem-Rubinow E, et al. Admission Glucose Levels and the Risk of Acute Kidney Injury in Nondiabetic ST Segment Elevation Myocardial Infarction Patients Undergoing Primary Percutaneous Coronary Intervention. Cardiorenal Med 2015;5:191-8.

34. Song JW, Shim JK, Yoo KJ, et al. Impact of intraoperative hyperglycaemia on renal dysfunction after off-pump coronary artery bypass Interact Cardiovasc Thorac Surg 2013;17:473-8.

35. Lane BR, Novick AC, Babineau D, et al. Comparison of Laparoscopic and Open Partial Nephrectomy for Tumor in a Solitary Kidney. J Urol 2008;179:847-51.

36. Thompson RH, Lane BR, Lohse CM, et al. Every Minute Counts When the Renal Hilum Is Clamped During Partial Nephrectomy. Eur Urol 2010;58:340-5.

37. Antonelli A, Cindolo L, Sandri M, et al. Predictors of the Transition from Off to On Clamp Approach during Ongoing Robotic Partial Nephrectomy: Data from the CLOCK Randomized Clinical Trial. J Urol 2019;202:62-8.

38. Cindolo L, Antonelli A, Sandri M, et al. The role of vascular clamping during robot-assisted partial nephrectomy for localized renal cancer: rationale and design of the CLOCK randomized phase III study. Minerva Urol Nefrol 2019;71:96-100.

39. Klatte T, Ficarra V, Gratzke C, et al. A Literature Review of Renal Surgical Anatomy and Surgical Strategies for 
Partial Nephrectomy. Eur Urol 2015;68:980-92.

40. Shen Y, Zhang W, Cheng X, et al. Association between postoperative fluid balance and acute kidney injury in patients after cardiac surgery: A retrospective cohort study. J Crit Care 2018;44:273-7.

41. Chen T, Guestrin C. XGBoost: A Scalable Tree Boosting

Cite this article as: Zhu $\mathrm{K}$, Song $\mathrm{H}$, Zhang Z, Ma B, Bao X, Zhang Q, Jin J. Acute kidney injury in solitary kidney patients after partial nephrectomy: incidence, risk factors and prediction. Transl Androl Urol 2020;9(3):1232-1243. doi:10.21037/ tau.2020.03.45
System. 2016.

42. Christodoulou E, Ma J, Collins GS, et al. A systematic review shows no performance benefit of machine learning over logistic regression for clinical prediction models. J Clin Epidemiol 2019;110:12-22. 